\title{
787 NATURAL KILLER CELL ENGAGERS ACTIVATE INNATE AND ADAPTIVE IMMUNITY AND SHOW SYNERGY WITH PROINFLAMMATORY CYTOKINES
}

Katrina Bykova*, Matthew Faber, Ke Liu, Noor Siddiqi, Matthew Bernett, Christine Bonzon, Juan Diaz, Dong Hyun Nam, Kendra Avery, Jing Qi, Rumana Rashid, Rena Bahjat, John Desjarlais. Xencor, Monrovia, CA, United States

Background Natural Killer cell Engagers (NKEs) are multifunctional molecules that target activating receptors on the surface of NK cells, bind to tumor associated antigens and engage Fc gamma receptors expressed on effector cells of the immune system. NKEs promote tumor cell lysis by redirecting NK cells to their targets, and drive activation and proliferation of NK cells. Engagement of NK cells, an effector cell population of the innate immune system, provides an opportunity to target cancers with reduced expression of MHC molecules that are less responsive to therapies targeting the adaptive immune system. Therefore, NKEs have a potential to provide an additional treatment option to patients who respond poorly to $\mathrm{T}$ cell tailored immunotherapies.

Methods Expanding on Xencor's XmAb bispecific Fc platform, we developed NKE molecules targeting NKG2D, an activating receptor expressed on cytotoxic immune cells, $\mathrm{B} 7 \mathrm{H} 3$, a pan tumor antigen, while simultaneously engaging $\mathrm{Fc}$ gamma receptors. Functional activity of NKEs was evaluated via assessing anti-tumor cytotoxicity and activation of NK and T cells in co-culture studies with human cancer cell lines.

Results NKEs were engineered for synergistic effects on NK cells by the simultaneous engagement of NKG2D and Fc gamma receptors. Additionally, the NKG2D variable domains were selected for their ability to provide a co-stimulatory signal to $\mathrm{T}$ cells in the presence of TCR-mediated signaling. Developed NKEs showed cytotoxic activity and immune cell activation in co-culture studies of human cancer cell lines with either PBMCs, $\mathrm{T}$ cells or NK cells. Combination of NKEs with proinflammatory cytokines, such as IL15, showed enhancement of the cytotoxic activity against tumor cells and augmented NK cell activation.

Conclusions $\mathrm{XmAb}$ bispecific NKEs engineered to engage innate and adaptive immunity show encouraging tumor cell killing activity and synergistic cytotoxicity in combination with proinflammatory cytokines. These data have identified several promising candidate NKEs for future in vivo efficacy studies in mouse tumor models expressing $\mathrm{B} 7 \mathrm{H} 3$.

http://dx.doi.org/10.1136/jitc-2021-SITC2021.787 